\title{
Modification and Validation of the Phosphate Removal Model: A Multicenter Study
}

\author{
Weichen Zhang ${ }^{\mathrm{a}}$ Qiuna Du ${ }^{\mathrm{b}}$ Jing Xiao ${ }^{c}$ Zhaori Bid ${ }^{\mathrm{d}}$ Chen $\mathrm{Yu}^{\mathrm{b}}$ Zhibin $\mathrm{Ye}^{\mathrm{c}}$ \\ Mengjing Wang ${ }^{a}$ Jing Chen ${ }^{a, d}$

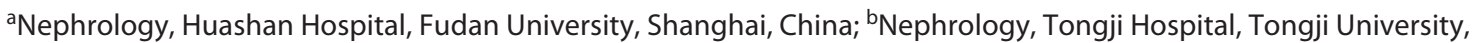

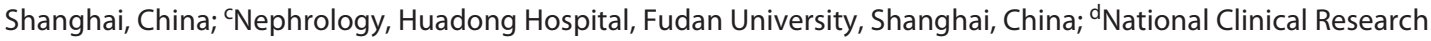 \\ Center for Aging and Medicine, Huashan Hospital, Fudan University, Shanghai, China
}

\section{Keywords}

Hemodialysis · Hyperphosphatemia · Phosphate removal ·

Model modification

\begin{abstract}
Background: Our research group has previously reported a noninvasive model that estimates phosphate removal within a 4-h hemodialysis (HD) treatment. The aim of this study was to modify the original model and validate the accuracy of the new model of phosphate removal for HD and hemodiafiltration (HDF) treatment. Methods: A total of 109 HD patients from 3 HD centers were enrolled. The actual phosphate removal amount was calculated using the area under the dialysate phosphate concentration time curve. Model modification was executed using second-order multivariable polynomial regression analysis to obtain a new parameter for dialyzer phosphate clearance. Bias, precision, and accuracy were measured in the internal and external validation to determine the performance of the modified model. Results: Mean age of the enrolled patients was
\end{abstract}

Weichen Zhang, Qiuna Du, and Jing Xiao contributed equally.

karger@karger.com www.karger.com/kbr

Karger $\stackrel{\text { ' }}{5}$

GOPEN ACCESS
(C) 2021 The Author(s)

Published by S. Karger AG, Basel

This article is licensed under the Creative Commons AttributionNonCommercial-NoDerivatives 4.0 International License (CC BYNC-ND) (http://www.karger.com/Services/OpenAccessLicense). Usage and distribution for commercial purposes as well as any distribution of modified material requires written permission.
$63 \pm 12$ years, and 67 (61.5\%) were male. Phosphate removal was $19.06 \pm 8.12 \mathrm{mmol}$ and $17.38 \pm 6.75 \mathrm{mmol}$ in 4-h HD and HDF treatments, respectively, with no significant difference. The modified phosphate removal model was expressed as $\mathrm{Tpo}_{4}=80.3 \times C_{45}-0.024 \times$ age $+0.07 \times$ weight $+\beta \times$ clearance $-8.14\left(\beta=6.231 \times 10^{-3} \times\right.$ clearance -1.886 $\times 10^{-5} \times$ clearance $\left.^{2}-0.467\right)$, where $C_{45}$ was the phosphate concentration in the spent dialysate measured at the 45th minute of HD and clearance was the phosphate clearance of the dialyzer. Internal validation indicated that the new model was superior to the original model with a significantly smaller bias and higher accuracy. External validation showed that $R^{2}$, bias, and accuracy were not significantly different than those of internal validation. Conclusions: $A$ new model was generated to quantify phosphate removal by 4-h HD and HDF with a dialyzer surface area of 1.3-1.8 $\mathrm{m}^{2}$. This modified model would contribute to the evaluation of phosphate balance and individualized therapy of hyperphosphatemia.

(C) 2021 The Author(s)

Published by S. Karger AG, Basel

Mengjing Wang

Division of Nephrology, Huashan Hospital, Fudan University

12 Middle Wurumuqi Road, Shanghai 200040 (China)

fiyona27@126.com or

Jing Chen

Division of Nephrology, National Clinical Research Center for Aging and Medicine Huashan Hospital, Fudan University

12 Middle Wurumuqi Road, Shanghai 200040 (China)

chenjing1998@fudan.edu.cn 


\section{Introduction}

Hyperphosphatemia is a common complication that leads to severe morbidity and poor prognosis in maintenance hemodialysis (MHD) patients. It is reported that increased serum phosphorus levels are associated with pruritus [1], vascular calcification [2], and left ventricular hypertrophy [3]. Evidence demonstrates that each 1-mg/ $\mathrm{dL}$ increase in serum phosphorus causes a higher risk of all-cause mortality by $18 \%$ [4] and cardiovascular mortality by $67 \%$ [5]. Despite prescribed treatment with phosphate binders, the prevalence of hyperphosphatemia in HD patients remains high, ranging from 40 to $60 \%$ according to the DOPPS annual report [6]. Thus, the control of hyperphosphatemia in MHD patients is far from satisfactory, especially in developing countries $[7,8]$. Phosphate homeostasis in dialysis patients with stable bone metabolism depends on phosphate intake, phosphate removal by HD, and phosphate binding by phosphate binders. Failure to achieve normal serum phosphate is mainly due to an increase in intake or a decrease in removal [9]. Thus, evaluation of phosphate balance is necessary to control hyperphosphatemia. Recently, the Renal Nutrition work group of the Kidney Disease Outcomes Quality Initiative (KDOQI) Guideline [10] emphasized the necessity of individualized treatment based on patient's need and clinical judgment rather than suggesting specific dietary phosphate ranges, making individual phosphate balance assessment critical in achieving adequate control of hyperphosphatemia in patients with end-stage renal disease.

Phosphate retention is calculated by phosphate absorption minus phosphate removal. Dietary phosphate intake of dialysis patients can be evaluated in various ways, such as the Food Frequency Questionnaire (FFQ) [11], 24-h dietary recalls [12], and 3-day food records $[13,14]$. The binding amount of phosphate by phosphate binders can be calculated according to the phosphate binding capacity per tablet and the dose of phosphate binders [15]. However, the quantification of phosphate removal by HD is not well understood.

Our research group has previously reported a noninvasive phosphate removal model that estimates phosphate removal within a 4-h HD treatment by using age, dry body weight, dialyzer phosphate clearance, and phosphate concentration in the spent dialysate measured $45 \mathrm{~min}$ after HD began [16]. In this predicting model, patients were prescribed LO PS 12 (B. Braun, Melsungen, Hessen, Germany) and Sureflux-130G (Nipro, Osaka, Japan) as the dialyzers for HD. However, newer dialyzers with higher phosphate clearance are available for MHD and hemodi- afiltration (HDF). Thus, the original model may be not applicable for the current HD or HDF treatments, necessitating model modification for further application. The aim of this study was to modify the original phosphate removal model of 4-h HD and validate the accuracy of the new model for HD and HDF treatment using different dialyzers among different HD centers.

\section{Materials and Methods}

This was a cross-sectional study conducted in 3 HD centers in Shanghai, including Huashan Hospital, Huadong Hospital, and Tongji Hospital, with the approval of the Ethics Committee on $\mathrm{Hu}$ man Research at each hospital (KY2018-470) and clinical trial registration at https://clinicaltrials.gov (NCT03861247). The study included 2 parts: first, modifying the parameters based on the original phosphate removal model for more dialyzers; second, validating the modified model by internal and external datasets.

\section{Study Population}

One hundred and nine patients undergoing 4-h HD sessions, 3 times per week using arteriovenous fistula or arteriovenous graft for $>3$ months, participated in this study after granting written, informed consent. The exclusion criteria were severe malnutrition, tumor, heart failure, cachexia, or active infection. Residual renal function was assumed as negligible during dialysis due to the limited 4-h session. Of the 109 patients, 74 patients from Huadong and Tongji hospitals were randomly divided into a modification group and an internal validation group in a 2:1 ratio as the dialyzers in these 2 hospitals included dialyzers used for original model creation. The remaining 35 patients who used different types of dialyzers from Huashan Hospital were used for external validation to quantify the predictive performance of the modified model [17].

\section{HD Dialyzers and Devices}

Both HD treatment and HDF treatment were included in this research. Patients used F14 (WEGO, Weihai, Shandong, China) for $\mathrm{HD}$ and $\mathrm{B}-16 \mathrm{H}$ and HIPS15 (B. Braun, Melsungen, Hessen, Germany) for HDF in Huashan Hospital, or FX8 (Fresenius, Homburg, Germany) for HD and FX80 (Fresenius, Homburg, Germany) for HDF in Huadong Hospital, or F15 (WEGO, Weihai, Shandong, China), Sureflux-150G (Nipro, Osaka, Japan), and REXEED-18LC (Asahi Kasei, Tokyo, Japan) for HD and FX800 (Fresenius, Homburg, Germany) and TS1.6 (TORAY, Tokyo, Japan) for HDF in Tongji Hospital. HD and HDF treatments were performed using the B. Braun Dialog + dialysis machine (Melsungen, Hessen, Germany), Gambro GBS13-AK95S dialysis machine (Baxter, Deerfield, IL, USA), or Fresenius 4008S dialysis machine (Homburg, Germany). The continuous online generation of bicarbonate-buffered dialysate with $\mathrm{Na}^{+} 135.0, \mathrm{HCO}_{3}{ }^{-} 35.0, \mathrm{Mg}^{2+} 0.5, \mathrm{~K}^{+} 2.0$, and $\mathrm{Ca}^{2+}$ $1.25-1.75 \mathrm{mmol} / \mathrm{L}$ was used. The dialysate did not contain glucose.

\section{Dialysate Collection and Phosphate Concentration}

Measurement

During the midweek HD and HDF treatment, 13 dialysate samples were collected from the outlet of the dialysis machine described in our previous study [16]. The blood flow rate was 243.95 
Table 1. Demographic and clinical characteristics of the modification group, internal validation group, and external validation group

\begin{tabular}{|c|c|c|c|c|}
\hline Characteristics & $\begin{array}{l}\text { Modification } \\
\text { group } \\
N=50\end{array}$ & $\begin{array}{l}\text { Internal } \\
\text { validation group } \\
N=24\end{array}$ & $\begin{array}{l}\text { External } \\
\text { validation group } \\
N=35\end{array}$ & $p$ value \\
\hline Male, $n(\%)$ & $34(68.0)$ & $16(66.7)$ & $17(48.6)$ & 0.163 \\
\hline Dialysis vintage, months & $83.23 \pm 53.12$ & $74.01 \pm 64.50$ & $114.10 \pm 80.95$ & 0.079 \\
\hline Dry body weight, kg & $64.86 \pm 16.08$ & $62.60 \pm 13.08$ & $57.07 \pm 10.97$ & $0.043^{\mathrm{a}}$ \\
\hline Glomerulonephritis & $20(40.0)$ & $8(33.3)$ & $18(51.4)$ & \multirow[t]{5}{*}{0.711} \\
\hline Diabetes & $13(26.0)$ & $6(25.0)$ & $4(11.4)$ & \\
\hline Hypertension & $8(16.0)$ & $3(12.5)$ & $4(11.4)$ & \\
\hline Polycystic kidney & $3(6.0)$ & $3(12.5)$ & $3(8.6)$ & \\
\hline Others & $6(12.0)$ & $4(16.7)$ & $6(17.2)$ & \\
\hline $\mathrm{HD} / \mathrm{HDF}, n / n$ & $31 / 19$ & $11 / 13$ & $21 / 14$ & 0.399 \\
\hline Calcium, mmol/L & $2.18 \pm 0.20$ & $2.25 \pm 0.17$ & $2.35 \pm 0.26$ & $0.02^{\mathrm{a}}$ \\
\hline Phosphorus, mmol/L & $1.78 \pm 0.54$ & $1.82 \pm 0.45$ & $1.56 \pm 0.49$ & $0.038^{\mathrm{a}}$ \\
\hline $\mathrm{iPTH}, \mathrm{pg} / \mathrm{mL}$ & $459.15 \pm 361.90$ & $337.16 \pm 247.72$ & $234.10 \pm 185.22$ & $0.004^{\mathrm{a}}$ \\
\hline ALP,$\mu / L$ & $127.62 \pm 114.17$ & $124.75 \pm 84.22$ & $85.27 \pm 21.41$ & 0.14 \\
\hline $\mathrm{Hb}, \mathrm{g} / \mathrm{L}$ & $105.66 \pm 12.50$ & $108.72 \pm 14.41$ & $108.80 \pm 15.13$ & 0.507 \\
\hline Alb, g/L & $39.04 \pm 3.41$ & $39.24 \pm 3.57$ & $40.66 \pm 2.98$ & $0.038^{\mathrm{a}}$ \\
\hline $\mathrm{BUN}, \mathrm{mmol} / \mathrm{L}$ & $27.85 \pm 6.23$ & $27.83 \pm 4.97$ & $26.65 \pm 6.11$ & 0.619 \\
\hline $\mathrm{SCr}, \mu \mathrm{mol} / \mathrm{L}$ & $944.83 \pm 218.32$ & $887.32 \pm 192.31$ & $915.54 \pm 194.40$ & 0.338 \\
\hline $\mathrm{UA}, \mathrm{mmol} / \mathrm{L}$ & $0.47 \pm 0.07$ & $0.44 \pm 0.12$ & $0.45 \pm 0.09$ & 0.202 \\
\hline Potassium, mmol/L & $4.75 \pm 0.81$ & $4.83 \pm 0.61$ & $4.71 \pm 0.605$ & 0.817 \\
\hline Dialyzer & $\begin{array}{l}\text { F15/SUREFLUX-150G/REXEED- } \\
\text { 18LC/FX8/FX80/FX800/TS1.6SL }\end{array}$ & $\begin{array}{l}\text { F15/SUREFLUX-150G/REXEED- } \\
\text { 18LC/FX8/FX80/FX800/TS1.6SL }\end{array}$ & F14/B-16H/HIPS15 & - \\
\hline
\end{tabular}

Values are expressed as mean $\pm \mathrm{SD}$ or number (percentage). Conversion factors for units: Alb and $\mathrm{Hb}$ in $\mathrm{g} / \mathrm{L}$ to $\mathrm{g} / \mathrm{dL}, \times 0.1 ; \mathrm{BUN}$ in $\mathrm{mmol} / \mathrm{L}$ to $\mathrm{mg} / \mathrm{dL}, \times 2.81$; $\mathrm{SCr}$ in $\mu \mathrm{mol} / \mathrm{L}$ to $\mathrm{mg} / \mathrm{dL}, \times 0.0113$; $\mathrm{UA}$ in $\mathrm{mmol} / \mathrm{L}$ to $\mathrm{mg} / \mathrm{dL}, \times 0.0168$; potassium in $\mathrm{mmol} / \mathrm{L}$ to $\mathrm{mg} / \mathrm{dL}, \times 3.91$; calcium in $\mathrm{mmol} / \mathrm{L}$ to $\mathrm{mg} / \mathrm{dL}, \times 4.0$; phosphorus in $\mathrm{mmol} / \mathrm{L}$ to $\mathrm{mg} / \mathrm{dL}, \times 3.1$; iPTH in $\mathrm{pg} / \mathrm{mL}$ to $\mathrm{pmol} / \mathrm{L}, \times 0.11$. No conversion is necessary for ALP. HD, hemodialysis; HDF, hemodiafiltration; iPTH, intact parathyroid hormone; ALP, alkaline phosphatase; Alb, albumin; $\mathrm{Hb}$, hemoglobin; BUN, blood urea nitrogen; SCr, serum creatinine; UA, uric acid. ${ }^{\mathrm{a}} p<0.05$ indicating significant difference among the modification group, internal validation group, and external validation group.

$\pm 19.20 \mathrm{~mL} / \mathrm{min}$ in HD treatment and $245.63 \pm 17.77 \mathrm{~mL} / \mathrm{min}$ in HDF treatment with no significant difference $(p=0.62)$. The dialysate was obtained with blood flows ranging from 180 to $300 \mathrm{~mL} /$ $\mathrm{min}$ and a convective dialysate flow rate of $500 \mathrm{~mL} / \mathrm{min}$ for $\mathrm{HD}$ and $500-700 \mathrm{~mL} / \mathrm{min}$ for HDF. All HDF treatments were executed using postdilution modality. We calculated the actual phosphate removal of 4-h dialysis using the area under the dialysate phosphate concentration time curve (AUC) since it was demonstrated to be as precise as the "gold standard" which asked for collecting all the spent dialysates [16]. Phosphate concentration was measured in each sample using the phosphomolybdate-based colorimetric method by an autoanalyzer (Hitachi 7600; Hitachi Co., Tokyo, Japan).

Rapid blood samples were drawn using uniform techniques for laboratory examination on the same day of dialysate collection. All laboratory values were measured using a Hitachi clinical chemistry analyzer (Tokyo, Japan) with standard laboratory techniques (performed by a technician in the biochemistry laboratory of Huashan Hospital).

\section{Modification of Original Phosphate Removal Model and Internal Validation}

The original phosphate removal model was expressed as follows [16]: $\mathrm{Tpo}_{4}=80.3 \times C_{45}-0.024 \times$ age $+0.07 \times$ weight +0.06 $\times$ clearance -8.14 , where $\mathrm{Tpo}_{4}$, given in $\mathrm{mmol} / 4 \mathrm{~h}$, was the predicted amount of phosphate removed in a dialysis session; $C_{45}$ was the effluent dialysate phosphate concentration at $45 \mathrm{~min}$ of treatment in $\mathrm{mmol} / \mathrm{L}$; age was the age of the patient in years; weight was the dry body weight of the patient in kg; clearance was the dialyzer phosphate clearance in in vitro conditions offered by the manufacturer's data sheet under the condition of $\mathrm{QB}=200 \mathrm{~mL} / \mathrm{min}$ and $\mathrm{QD}=500 \mathrm{~mL} / \mathrm{min}$. We assumed that original phosphate removal 


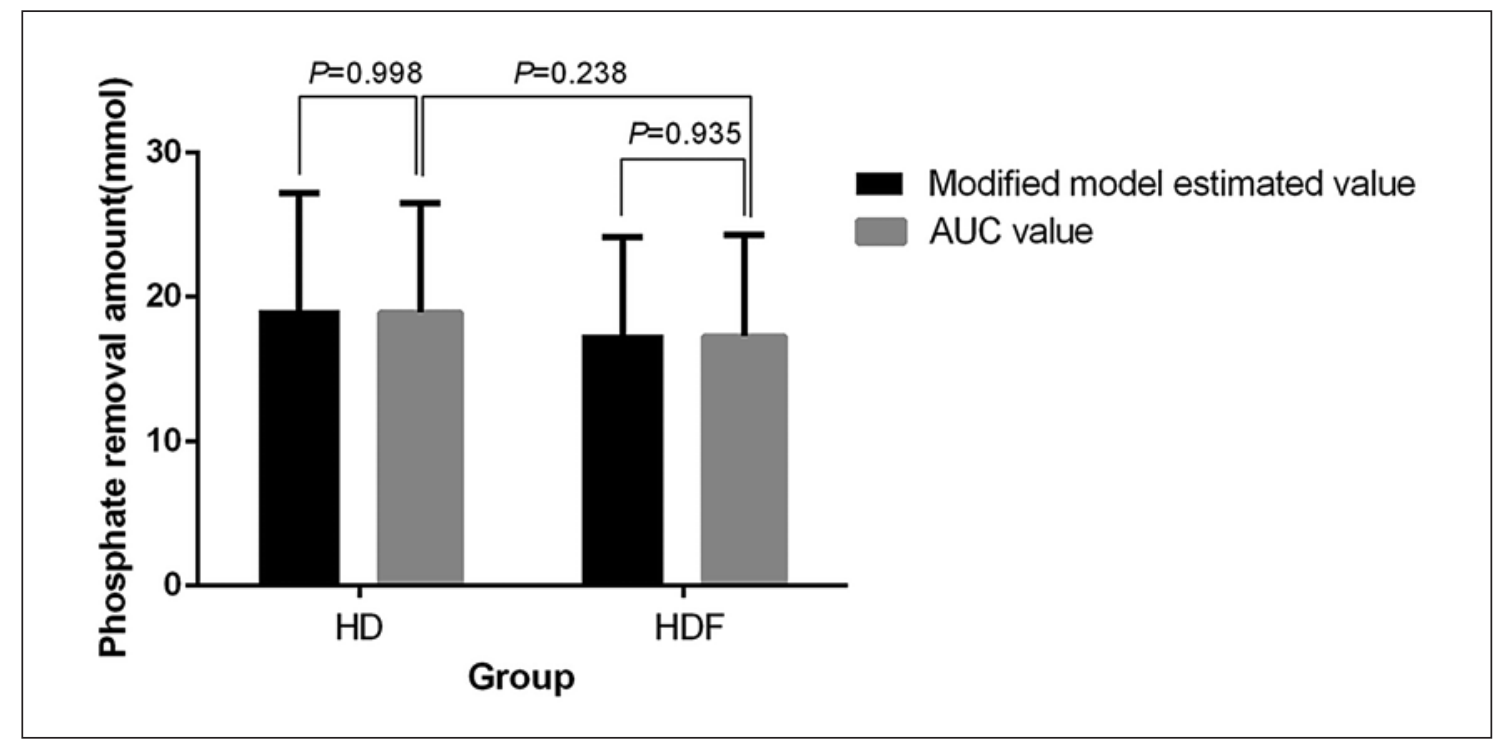

Fig. 1. Estimated value of phosphate removal by AUC (actual value) and modified model (estimated value) in 4-h $\mathrm{HD}$ and HDF. Actual phosphate removal per HD session calculated by AUC was $19.06 \pm 8.12 \mathrm{mmol}$ and 17.38 $\pm 6.75 \mathrm{mmol}$ in 4 -h HD and HDF, respectively. In addition, no significant difference was found between the AUC value and the modified model estimated value. HD, hemodialysis; HDF, hemodiafiltration.

model performance could be improved by modifying the coefficient of dialyzer phosphorus clearance as the main change of dialysis treatment was the dialyzer [18]. Correlation analysis showed that phosphate removal was not linearly correlated with dialyzer phosphate clearance. Thus, to improve estimation accuracy, the study introduced the multivariable polynomial regression method based on the Taylor theorem in mathematical analysis [19] to approximate the implicit complex nonlinear relationship between phosphate removal and dialyzer phosphate clearance. We used a second-order function as it met the requirement of estimation accuracy. In addition, the second-order function was convenient for clinical application. More specifically, we fixed the parameters of $C_{45}$, age, and weight and used second-order multivariable polynomial regression analysis to obtain a new parameter for dialyzer phosphate clearance.

\section{Internal and External Validation}

Bias, precision, and accuracy were measured to determine the performance of the original model and the modified model. Differences were calculated by subtracting AUC values from the estimated value of the model. Thus, a negative difference indicated that the model underestimated actual removal amount of phosphate. Bias was defined as the median difference. Precision was assessed as an interquartile range (IQR) of the differences. Accuracy was measured as the percentage of estimated value within 15 $\left(P_{15}\right), 30\left(P_{30}\right)$, and $50 \%\left(P_{50}\right)$ of the measured value and also as root mean square error (RMSE) $[18,20]$. In addition, $R^{2}$ was used to test the goodness of fit of the model, and it was aimed to be above 0.8 to guarantee precise estimation [21].

\section{Statistical Analysis}

Quantitative variables of patients' characteristics were expressed as mean \pm SD or as median (IQR), while categorical vari- ables were expressed as percentages or ratios. Differences were evaluated using the $\chi^{2}$ test, independent $t$ test, and Kruskal-Wallis test. Linear regression was used to describe the relationship between the estimated value of the modified model and the measurement value. Bland-Altman analysis was used to assess the agreement between the 2 measures of phosphate removal. All analyses were performed using SPSS version 19 (Chicago, IL, USA). A $p$ value of $<0.05$ was considered statistically significant.

\section{Results}

\section{General Data}

Mean age of the enrolled patients was $63 \pm 12$ years with an average dialysis vintage of $82 \pm 61$ months, and 67 (61.5\%) of them were male. Demographic, clinical, and laboratory data of the modification group, the internal validation group, and the external validation group are presented in Table 1. Patients from the modification group and internal validation group had higher dry body weight $(p=0.043)$, serum phosphorus $(p=0.038)$, and iPTH $(p=0.004)$ but had lower serum calcium $(p=0.02)$. Other characteristics were not significantly different among 3 groups. Dialyzers used in the 3 groups are shown in online suppl. Table 1 (for all online suppl. material, see www.karger.com/doi/10.1159/000511375) with phosphate clearance ranging from 158 to $192 \mathrm{~mL} / \mathrm{min}$ (online suppl. Table 1). 


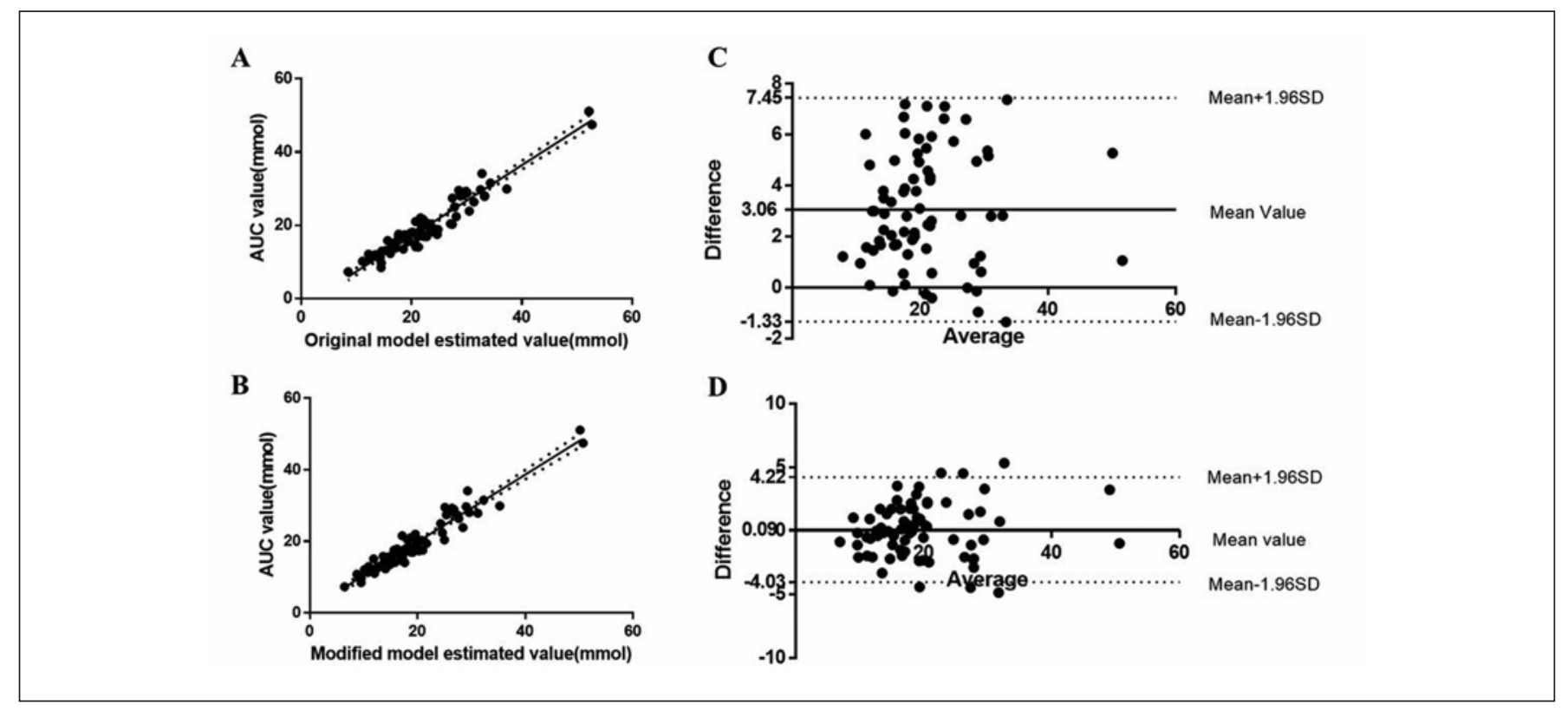

Fig. 2. Linear regression and Bland-Altman analysis using the internal validation dataset. A Linear regression analysis of the original model estimated value and the AUC value $\left(R^{2}=0.934, p<\right.$ 0.001). B Linear regression analysis of the modified model estimated value and the AUC value $\left(R^{2}=0.932, p<0.001\right)$. C BlandAltman plot of phosphate removal estimated by the AUC method and the original model (mean value of difference $=3.06, \mathrm{SD}=$ 2.24). Difference in the vertical axis stands for subtracting the AUC value from the original model estimated value. Average in the horizontal axis means average value of the AUC value and the model estimated value. D Bland-Altman plot of phosphate removal estimated by the AUC method and the modified model (mean value of difference $=0.09, \mathrm{SD}=2.11$ ). Difference in the vertical axis stands for subtracting the AUC value from the modified model estimated value. Average in the horizontal axis means average value of the AUC value and the modified model estimated value.

Table 2. Overall performance of the original model compared with the modified model by bias, precision, and accuracy using the internal validation dataset

\begin{tabular}{lccc}
\hline Parameters & Original model & Modified model & $p$ value \\
\hline Y-intercept (95\% CI) & $3.71(2.40-5.01)$ & $1.08(-0.18$ to 2.35$)$ & - \\
Slope (95\% CI) & $0.97(0.91-1.03)$ & $0.94(0.88-0.99)$ & - \\
$R^{2}$ & 0.934 & 0.932 & - \\
Bias, mmol & & & $<0.001^{\mathrm{a}}$ \\
$\quad$ Median of difference (IQR) & $2.81(1.48-4.95)$ & $-0.01(-1.11$ to -1.47$)$ & - \\
$\quad$ Precision, mmol & 3.47 & 2.58 & $0.023^{\mathrm{a}}$ \\
Accuracy, \% & & & $0.002^{\mathrm{a}}$ \\
$\quad P_{15}$ & 40 & 72 & 0.312 \\
$P_{30}$ & 60 & 96 & 0.91 \\
$P_{50}$ & 96 & 100 & 2.02 \\
$\quad$ RMSE & 2.2 & &
\end{tabular}

$P_{15}, P_{30}$, and $P_{50}$ stand for the percentage of the estimated value within $15,30,50 \%$ of the measured value. RMSE, root mean square error; CI, confidence interval; IQR, interquartile range. ${ }^{a} p<0.05$.

\section{Modification of Phosphate Removal Model}

Actual phosphate removal per HD session calculated by AUC was $19.06 \pm 8.12 \mathrm{mmol}$ and $17.38 \pm 6.75 \mathrm{mmol}$ in 4-h HD and HDF, respectively (Fig. 1). No significant difference was found between 2 dialysis modalities ( $p=$ 0.238 ). Therefore, the following modification was performed by mixing the HD and HDF treatment as a whole. A new variable coefficient $\beta$ was elicited by multivariable 
Table 3. External validation versus internal validation of the modified model by bias, precision, and accuracy

\begin{tabular}{lccc}
\hline Parameters & Internal validation & External validation & $p$ value \\
\hline Y-intercept (95\% CI) & $1.08(-0.18$ to 2.35$)$ & $0.45(-1.97$ to 2.88$)$ & - \\
Slope (95\% CI) & $0.94(0.88-0.99)$ & $0.97(0.83-1.12)$ & - \\
$R^{2}$ & 0.932 & 0.851 & - \\
Bias, mmol & & & 0.384 \\
$\quad$ Median of difference (IQR) & $-0.01(-1.11$ to 1.47$)$ & $-0.24(-0.68$ to 1.64$)$ & - \\
$\quad$ Precision, mmol & 2.58 & 2.32 & 0.47 \\
Accuracy, $\%$ & 72 & 80 & 0.764 \\
$\quad P_{15}$ & 96 & 94 & - \\
$P_{30}$ & 100 & 100 & 0.733 \\
$P_{50}$ & 2.02 & 1.64 & \\
RMSE & & & \\
\hline
\end{tabular}

$P_{15}, P_{30}$, and $P_{50}$ stand for the percentage of the estimated value within $15,30,50 \%$ of the measured value. No significant differences $(p<0.05)$ were observed between internal validation and external validation data. RMSE, root mean square error; $\mathrm{CI}$, confidence interval; IQR, interquartile range.

polynomial regression on the basis of the original model (model 1). The new model was then expressed as the following model 2:

$$
\begin{aligned}
& \mathrm{Tpo}_{4}=80.3 \times \mathrm{C}_{45}-0.024 \times \text { age }+0.07 \times \text { weight }+ \\
& 0.06 \times \text { clearance }-8.14 \\
& \mathrm{Tpo}_{4}=80.3 \times \mathrm{C}_{45}-0.024 \times \text { age }+0.07 \times \text { weight }+ \\
& \beta \times \text { clearance }-8.14 \\
& \beta=6.231 \times 10^{-3} \times \text { clearance }-1.886 \times 10^{-5} \times \\
& \text { clearance }{ }^{2}-0.467
\end{aligned}
$$

\section{Internal Validation}

The performance of the modified model and the original model in the internal validation dataset is further explored in Table 2 and Figure 2. Internal validation indicated the appropriateness of the modified model with an $R^{2}$ of 0.932 and RMSE of 2.02. The modified model fits the data better with significantly lower bias $(-0.01 \mathrm{mmol}$ of the modified model vs. $2.81 \mathrm{mmol}$ of the original model) and higher $P_{15}$ (72\% of the modified model vs. $40 \%$ of the original model) and $P_{30}(96 \%$ of the modified model vs. $60 \%$ of the original model) compared to the original model (Table 2; Fig. 2A, B). Other parameters such as $R^{2}$, RMSE, and $P_{50}$ were similar between the 2 models. Furthermore, the Bland-Altman plot demonstrated less bias in the new model with a mean difference of 0.09 and $95 \%$ confidence interval of -4.03 to 4.22 , while the original model had a mean difference of 3.06 and a $95 \%$ confidence interval of -1.33 to 7.45 (Fig. 2C, D).

\section{External Validation}

External validation versus internal validation of the modified model is presented in Table $3 . R^{2}$ of the modified model was lower in external validation $\left(R^{2}=0.851\right)$ compared to internal validation $\left(R^{2}=0.932\right)$ but was still within the acceptable range $[18,21]$ (Fig. 3A). The BlandAltman plot showed that the mean difference of external validation was -0.25 with a $95 \%$ confidence interval of -3.30 to 3.80 (Fig. 3B). Bias in external validation was lower than that in internal validation, at $-0.24 \mathrm{mmol}$; however, no significant difference was found between them. Precision was $2.32 \mathrm{mmol}$ in the external validation which was also similar to that in internal validation. Accuracy analysis showed that $P_{15}, P_{30}, P_{50}$, and RMSE in external validation performed as well as those in internal validation, indicating that the modified model was stable and accurate in estimating the HD phosphate removal when using different dialyzers.

Meanwhile, after we compared the difference in bias, precision, and accuracy of the modified model when applied to HD as compared to HDF using the whole cohort $(N=109)$, we found that there was no significant difference in the above parameters between HD and HDF (online suppl. Table 2; online suppl. Fig. 1).

\section{Discussion}

In the present study, we obtained a new model of quantifying phosphate removal by using data from various HD centers and types of dialyzers. Internal validation 


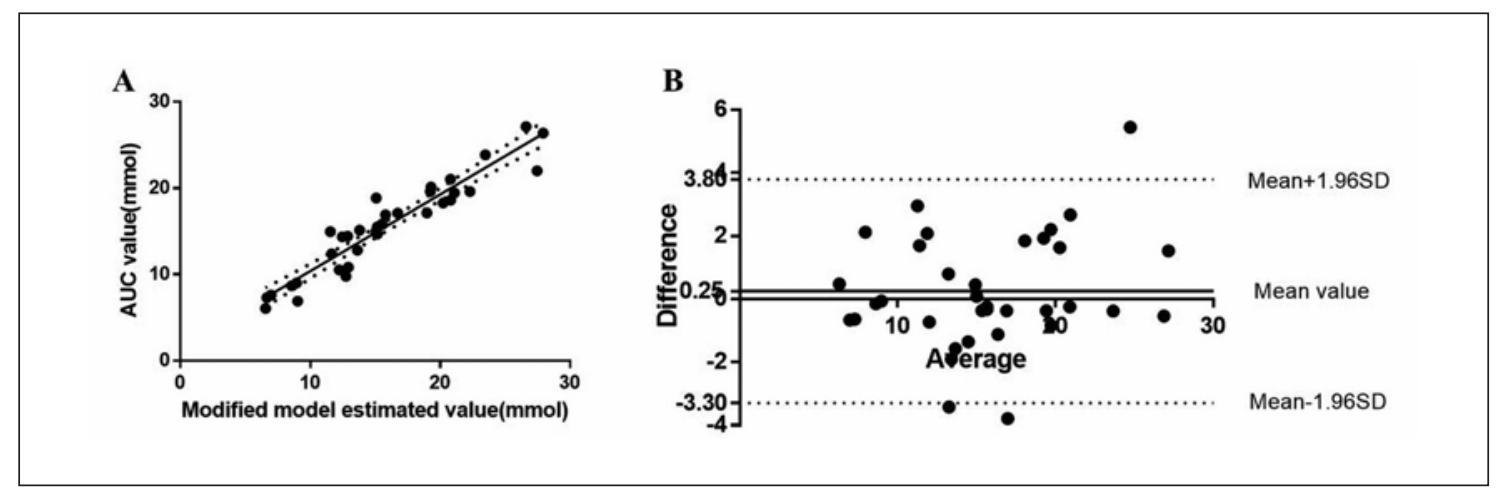

Fig. 3. Linear regression and Bland-Altman analysis using the external validation dataset. A Linear regression analysis of the modified model estimated value and the AUC value $\left(R^{2}=0.851, p<0.001\right)$. B Bland-Altman plot of phosphate removal estimated by the AUC method and the modified model (mean value of difference $=0.25$, $\mathrm{SD}=1.81$ ). Difference in the vertical axis stands for subtracting the AUC value from the modified model estimated value. Average in the horizontal axis means average value of the AUC value and the modified model estimated value.

showed that the modified model had lower bias and higher $P_{15}$ and $P_{30}$ than the original model. External validation showed that bias, precision, and accuracy were equivalent in external validation when compared to internal validation. Such results suggested that the modified model performed better than the previous model when estimating phosphate removal in both HD and HDF modality using a variety of dialyzers.

With the increasing emphasis on the precise management of hyperphosphatemia, estimation of phosphorus intake and excretion has assumed greater importance in $\mathrm{HD}$ patients [22]. According to the KDIGO guidelines, phosphorus-restricted diet, phosphate binders, and dialysis are recommended treatments for hyperphosphatemia [23]. Dialysis is the major approach for phosphate elimination, especially for patients without residual renal function. Numerous studies have pointed out that intensive HD and nocturnal HD lead to better phosphate removal than conventional HD and may improve the prognosis of patients [24-26]. However, such a dialysis modality is not available for most of the dialysis centers, especially in developing countries. Thus, quantification of phosphate removal becomes important as it allows physicians to have a better understanding of phosphate balance in HD patients and treat them individually.

Some evidence showed that phosphate removal may be influenced by various factors such as age, duration of dialysis session, membrane type, predialysis serum phosphate levels, serum PTH level, vitamin D administration, and different dialysis modalities [27-30]. Other studies also found that membrane type or dialysis modality were not related to phosphate removal [31-33]. This paradox may be caused by the diversity of clinical features in different dialysis centers, demonstrating the necessity of multicenter research. To our knowledge, this is the first multicenter study of model construction to evaluate phosphate removal by HD and HDF. Our study indicated that age, dry body weight, dialyzer phosphate clearance, and dialysate phosphate concentration at $45 \mathrm{~min}$ into treatment were the main factors that correlated with dialysis phosphorus removal. Some researchers such as Argiles et al. [34] and Gutzwiller et al. [35] preferred serum phosphorus level and $\mathrm{Kt} / \mathrm{V}$ as parameters for a predictive model. In contrast, this modified model chose noninvasive parameters to evaluate phosphate removal, making it more acceptable for clinical practice.

Previous studies demonstrated that serum phosphorus level [36] and phosphate diffusion [31] were related to dialysis phosphate removal. In our model, the coefficient of $C_{45}$ and $C_{45}$ itself may reflect not only the difference in phosphorus level but also the phosphate diffusion [16] under different physiological conditions of patients. This may also explain why the modified model estimated phosphate removal without large bias even when dry body weight, serum calcium, phosphorus, $\mathrm{iPTH}$, and albumin were different among the modification group, internal validation group, and external validation group.

In this study, we found phosphate removal by HD treatment was similar to HDF treatment. There were also studies indicating that the amount of phosphate removed was not significantly different between HD and HDF [37-39]. However, some previous studies demonstrated that phos- 
phate removal by HDF was higher than HD. Such as, Minutolo et al. [40] found that phosphate removal by HDF was greater than HD in the presence of similar predialytic serum phosphorus levels. Pedrini et al. [41] and other investigators [42-45] demonstrated that online HDF showed greater efficiency than low-flux HD in removing serum phosphate. Several mechanisms may explain the opposite results. First, phosphate removal is mainly correlated with the predialytic serum phosphorus level and dialyzer surface area $[30,46,47]$. In the studies mentioned above, predialytic serum phosphorus levels are not significantly different between the HD and HDF groups while dialyzer surface area is much larger in the HDF group. However, dialyzer surface area was 1.4-1.8 $\mathrm{m}^{2}$ in the HD group and 1.5-1.8 $\mathrm{m}^{2}$ in the HDF group in our study. Similar dialyzer surface area likely leads to undifferentiated phosphate removal quantities. Second, the molecular weight of the phosphate anion is $95 \mathrm{Da}$, and the clearance is mainly caused by diffusion [48]. The contribution of convection in HDF may be limited on phosphorus clearance [49]. In addition, dialyzer phosphate clearance in our model reflects the effect of the dialyzer membrane and surface area to some extent. Therefore, this modified model is applicable for HD and HDF dialyzers with different surface areas.

Our study has several limitations. Firstly, the modified model needs further external validation in a larger number of patients undergoing HD and HDF treatment using dialyzers that have a surface area above $1.8 \mathrm{~m}^{2}$. Secondly, the model was established and modified only from patients who underwent HD treatment for $4 \mathrm{~h}$ per session. The quantification of phosphate removal in long-time $\mathrm{HD}$ and HDF treatment remains for future study. Thirdly, the model was created and modified in postdiluted $\mathrm{HDF}$, and it should be further validated in a predilution modality. Finally, the study was done only in the Chinese population. Further studies will need to be conducted in other ethnic groups.

In conclusion, this study reported a new phosphate removal model of 4-h HD by modification, internal, and external validation of the phosphate removal model we generated before. It would help clinicians to evaluate the phosphate balance in patients undergoing HD and HDF treatment as well as explore individualized treatment strategies.

\section{Acknowledgements}

We thank the staff and participants from the Huashan, Huadong, and Tongji hospitals for providing the clinical data for this research.

\section{Statement of Ethics}

This study was conducted according to the guidelines laid down in the Declaration of Helsinki, and all procedures involving human subjects were approved by the Ethics Committee of Huashan, Huadong, and Tongji hospitals. Written informed consent was taken from the participants.

\section{Conflict of Interest Statement}

The authors declare that they have no relevant financial interest.

\section{Funding Sources}

This work was financially supported by the Shanghai Shenkang Hospital Development Center Clinical Science and Technology Innovation Project (No. SHDC12018111, Jing Chen; No. SHDC2020CR4014, Mengjing Wang), Shanghai Municipal Science and Technology Commission researching fund (No. 17411950701; Jing Chen), Shanghai Medical Leading Talents Project (No. 2019LJ03; Jing Chen), China Natural Science Grant (Nos. 81730017 and 81570665, Jing Chen; No. 81600577, Mengjing Wang), China National Key Research and Development Program (No. 2020YFC2005003, Jing Chen) and Shanghai Natural Science Grant (No. 16ZR1449400; Mengjing Wang). The funders had no role in study design; collection, analysis, and interpretation of data; writing the report; or the decision to submit the final report for publication.

\section{Author Contributions}

Research idea and study design: Weichen Zhang, Mengjing Wang, and Jing Chen; data acquisition: Weichen Zhang, Jing Xiao, and Qiuna Du; statistical analysis: Weichen Zhang, Zhaori Bi, Mengjing Wang, Jing Xiao, and Qiuna Du; supervision or mentorship: Chen Yu, Zhibin Ye, and Jing Chen.

\section{References}

1 Rayner HC, Larkina M, Wang M, GrahamBrown M, van der Veer SN, Ecder T, et al. International comparisons of prevalence, awareness, and treatment of pruritus in people on hemodialysis. Clin J Am Soc Nephrol. 2017;12:2000-7.

2 Nishizawa Y, Jono S, Ishimura E, Shioi A. Hyperphosphatemia and vascular calcification in end-stage renal disease. J Ren Nutr. 2005; 15(1):178-82.

3 Achinger SG, Ayus JC. Left ventricular hypertrophy: is hyperphosphatemia among dialysis patients a risk factor? J Am Soc Nephrol. 2006; 17(12 Suppl 3):S255-61. DOI: $10.1159 / 000511375$ 
4 Palmer SC, Hayen A, Macaskill P, Pellegrini F, Craig JC, Elder GJ, et al. Serum levels of phosphorus, parathyroid hormone, and calcium and risks of death and cardiovascular disease in individuals with chronic kidney disease: a systematic review and meta-analysis. JAMA. 2011;305(11):1119-27.

5 Rivara MB, Ravel V, Kalantar-Zadeh K, Streja E, Lau WL, Nissenson AR, et al. Uncorrected and albumin-corrected calcium, phosphorus, and mortality in patients undergoing maintenance dialysis. J Am Soc Nephrol. 2015;26(7): 1671-81.

6 Liabeuf S, McCullough K, Young EW, Pisoni $\mathrm{R}$, Zee J, Reichel H, et al. International variation in the management of mineral bone disorder in patients with chronic kidney disease: results from CKDopps. Bone. 2019;129: 115058.

7 Afifi A, El-Sayed H, El-Setouhi M, Ahmed H, Khalifa N. Hyperphosphatemia among endstage renal disease patients in developing countries: a forgotten issue? Hemodial Int. 2005;9(4):409-15.

8 Kong X, Zhang L, Zhang L, Chen N, Gu Y, Yu $\mathrm{X}$, et al. Mineral and bone disorder in Chinese dialysis patients: a multicenter study. BMC Nephrol. 2012;13:116.

9 Rivara MB, Ravel V, Streja E, Obi Y, Soohoo $\mathrm{M}$, Cheung AK, et al. Weekly standard Kt/ Vurea and clinical outcomes in home and incenter hemodialysis. Clin J Am Soc Nephrol. 2018;13(3):445-55.

10 Isakova T, Nickolas TL, Denburg M, Yarlagadda S, Weiner DE, Gutiérrez OM, et al. KDOQI US commentary on the 2017 KDIGO clinical practice guideline update for the diagnosis, evaluation, prevention, and treatment of chronic kidney disease-mineral and bone disorder (CKD-MBD). Am J Kidney Dis. 2017;70:737-51.

11 Noori N, Kalantar-Zadeh K, Kovesdy CP, Bross R, Benner D, Kopple JD. Association of dietary phosphorus intake and phosphorus to protein ratio with mortality in hemodialysis patients. Clin J Am Soc Nephrol. 2010;5(4): 683-92.

12 Chang AR, Lazo M, Appel LJ, Gutiérrez OM, Grams ME. High dietary phosphorus intake is associated with all-cause mortality: results from NHANES III. Am J Clin Nutr. 2014; 99(2):320-7.

13 Shinozaki N, Murakami K, Asakura K, Uechi K, Kobayashi S, Masayasu S, et al. Dietary phosphorus intake estimated by 4-day dietary records and two 24-hour urine collections and their associated factors in Japanese adults. Eur J Clin Nutr. 2018;72(4):517-25.

14 Martins AM, Dias Rodrigues JC, de Oliveira Santin FG, Barbosa Brito Fd S, Bello Moreira AS, Lourenço RA, et al. Food intake assessment of elderly patients on hemodialysis. J Ren Nutr. 2015;25(3):321-6.

15 Daugirdas JT, Finn WF, Emmett M, Chertow GM. The phosphate binder equivalent dose. Semin Dial. 2011;24(1):41-9.
16 Wang M, Li H, Liao H, Yu Y, You L, Zhu J, et al. Phosphate removal model: an observational study of low-flux dialyzers in conventional hemodialysis therapy. Hemodial Int. 2012; 16(3):363-76.

17 Moons KG, Kengne AP, Grobbee DE, Royston P, Vergouwe Y, Altman DG, et al. Risk prediction models: II. External validation, model updating, and impact assessment. Heart. 2012;98(9):691-8.

18 Ma YC, Zuo L, Chen JH, Luo Q, Yu XQ, Li Y, et al. Modified glomerular filtration rate estimating equation for Chinese patients with chronic kidney disease. J Am Soc Nephrol. 2006;17(10):2937-44.

19 Wei J, Chen T, Liu G, Yang J. Higher-order multivariable polynomial regression to estimate human affective states. Sci Rep. 2016;6: 23384.

20 Kilbride HS, Stevens PE, Eaglestone G, Knight S, Carter JL, Delaney MP, et al. Accuracy of the MDRD (modification of diet in renal disease) study and CKD-EPI (CKD epidemiology collaboration) equations for estimation of GFR in the elderly. Am J Kidney Dis. 2013; 61(1):57-66.

21 Stevens LA, Schmid CH, Zhang YL, Coresh J, Manzi J, Landis R, et al. Development and validation of GFR-estimating equations using diabetes, transplant and weight. Nephrol Dial Transplant. 2010;25(2):449-57.

22 Copland M, Komenda P, Weinhandl ED, McCullough PA, Morfin JA. Intensive hemodialysis, mineral and bone disorder, and phosphate binder use. Am J Kidney Dis. 2016; 68(5S1):S24-32.

23 Isakova T, Nickolas TL, Denburg M, Yarlagadda S, Weiner DE, Gutiérrez OM, et al. KDOQI US commentary on the 2017 KDIGO clinical practice guideline update for the diagnosis, evaluation, prevention, and treatment of chronic kidney disease-mineral and bone disorder (CKD-MBD). Am J Kidney Dis. 2017;70:737-51.

24 Ayus JC, Achinger SG, Mizani MR, Chertow GM, Furmaga W, Lee S, et al. Phosphorus balance and mineral metabolism with $3 \mathrm{~h}$ daily hemodialysis. Kidney Int. 2007;71(4):336-42.

25 Culleton BF, Walsh M, Klarenbach SW, Mortis G, Scott-Douglas N, Quinn RR, et al. Effect of frequent nocturnal hemodialysis vs conventional hemodialysis on left ventricular mass and quality of life: a randomized controlled trial. JAMA. 2007;298(11):1291-9.

26 Ayus JC, Mizani MR, Achinger SG, Thadhani $\mathrm{R}$, Go AS, Lee S. Effects of short daily versus conventional hemodialysis on left ventricular hypertrophy and inflammatory markers: a prospective, controlled study. J Am Soc Nephrol. 2005;16(9):2778-88.

27 Albalate M, de la Piedra C, Fernández C, Lefort M, Santana H, Hernando P, et al. Association between phosphate removal and markers of bone turnover in haemodialysis patients. Nephrol Dial Transplant. 2006; 21(6):1626-32.
28 Ratanarat R, Brendolan A, Volker G, Bonello M, Salvatori G, Andrikos E, et al. Phosphate kinetics during different dialysis modalities. Blood Purif. 2004;23(1):83-90.

29 Sampaio MS, Ruzany F, Dorigo DM, Suassuna JH. Phosphate mass removal during hemodialysis: a comparison between eKt/V-matched conventional and extended dialysis. Am J Nephrol. 2012;36(2):121-6.

30 Tonelli M, Wang W, Hemmelgarn B, Lloyd A, Manns B. Phosphate removal with several thrice-weekly dialysis methods in overweight hemodialysis patients. Am J Kidney Dis. 2009; 54(6):1108-15.

31 DeSoi CA, Umans JG. Phosphate kinetics during high-flux hemodialysis. J Am Soc Nephrol. 1993;4(5):1214-8.

32 Katopodis KP, Chala A, Koliousi E, Takouli L, Kalaitzidis R, Theodorou J, et al. Role of the dialyzer membrane on the overall phosphate kinetics during hemodialysis. Blood Purif. 2005;23(5):359-64.

33 Thompson S, Manns B, Lloyd A, Hemmelgarn B, MacRae J, Klarenbach S, et al. Impact of using two dialyzers in parallel on phosphate clearance in hemodialysis patients: a randomized trial. Nephrol Dial Transplant. 2017;32(5):855-61.

34 Argiles A, Ficheux A, Thomas M, Bosc JY, Kerr PG, Lorho R, et al. Precise quantification of dialysis using continuous sampling of spent dialysate and total dialysate volume measurement. Kidney Int. 1997;52:530-7.

35 Gutzwiller JP, Schneditz D, Huber AR, Schindler C, Gutzwiller F, Zehnder CE. Estimating phosphate removal in haemodialysis: an additional tool to quantify dialysis dose. Nephrol Dial Transplant. 2002;17(6):103744.

36 Gotch FA, Panlilio F, Sergeyeva O, Rosales L, Folden T, Kaysen G, et al. A kinetic model of inorganic phosphorus mass balance in hemodialysis therapy. Blood Purif. 2003;21(1):517.

37 Nagaoka Y, Matsumoto H, Okada T, Iwasawa $\mathrm{H}$, Tomaru R, Wada T, et al. Benefits of firsthalf intensive haemodiafiltration for the removal of uraemic solutes. Nephrology. 2011; 16(5):476-82.

38 Bellien J, Fréguin-Bouilland C, Joannidès R, Hanoy M, Rémy-Jouet I, Monteil C, et al. High-efficiency on-line haemodiafiltration improves conduit artery endothelial function compared with high-flux haemodialysis in end-stage renal disease patients. Nephrol Dial Transplant. 2014;29(2):414-22.

39 Jean G, Hurot JM, Deleaval P, Mayor B, Lorriaux C. Online-haemodiafiltration vs. conventional haemodialysis: a cross-over study. BMC Nephrol. 2015;16:70.

40 Minutolo R, Bellizzi V, Cioffi M, Iodice C, Giannattasio $P$, Andreucci M, et al. Postdialytic rebound of serum phosphorus: pathogenetic and clinical insights. J Am Soc Nephrol. 2002; 13(4):1046-54. 
41 Pedrini LA, De Cristofaro V, Comelli M, Casino FG, Prencipe M, Baroni A, et al. Longterm effects of high-efficiency on-line haemodiafiltration on uraemic toxicity. A multicentre prospective randomized study. Nephrol Dial Transplant. 2011;26(8):261724.

42 Maduell F, Arias M, Durán CE, Vera M, Fontseré $N$, Azqueta $M$, et al. Nocturnal, everyother-day, online haemodiafiltration: an effective therapeutic alternative. Nephrol Dial Transplant. 2012;27(4):1619-31.

43 Mandolfo S, Borlandelli S, Imbasciati E, Badalamenti S, Graziani G, Sereni L, et al. Pilot study to assess increased dialysis efficiency in patients with limited blood flow rates due to vascular access problems. Hemodial Int. 2008;12(1):55-61.

44 Mineshima M, Eguchi K. Development of intermittent infusion hemodiafiltration using ultrapure dialysis fluid with an automated dialysis machine. Blood Purif. 2013;35 Suppl 1: 55-8.

45 Lornoy W, De Meester J, Becaus I, Billiouw JM, Van Malderen PA, Van Pottelberge M. Impact of convective flow on phosphorus removal in maintenance hemodialysis patients. J Ren Nutr. 2006;16(1):47-53.

46 Leypoldt JK. Kinetics of beta2-microglobulin and phosphate during hemodialysis: effects of treatment frequency and duration. Semin Dial. 2005;18(5):401-8.

47 Kuhlmann MK. Phosphate elimination in modalities of hemodialysis and peritoneal dialysis. Blood Purif. 2010;29(2):137-44.

48 Daugirdas JT. Removal of phosphorus by hemodialysis. Semin Dial. 2015 Nov-Dec;28(6): 620-3.

49 Cornelis T, van der Sande FM, Eloot S, Cardinaels E, Bekers O, Damoiseaux J, et al. Acute hemodynamic response and uremic toxin removal in conventional and extended hemodialysis and hemodiafiltration: a randomized crossover study. Am J Kidney Dis. 2014;64(2): $247-56$ 